\title{
Digital image correlation method: a versatile tool for engineering and art structures investigations
}

\author{
M. Kujawinska ${ }^{1}$, M. Malesa ${ }^{1}$, K. Malowany ${ }^{1}$, A. Piekarczuk ${ }^{2}$, \\ L. Tymińska-Widmer ${ }^{3}$, P.Targowski ${ }^{4}$ \\ ${ }^{1}$ Warsaw University of Technology, Institute of Micromechanics and Photonics, 02-525 Warsaw, 8 Sw. A. Boboli St., \\ Poland \\ ${ }^{2}$ Building Research Institute, 00-611 Warsaw, 1 Filtrowa St., Poland \\ ${ }^{3}$ Institute for the Study, Restoration and Conservation of Cultural Heritage, Nicolaus Copernicus University, 7 Gagarina \\ St., 87-100 Toruń, Poland \\ ${ }^{4}$ Institute of Physics, Nicolaus Copernicus University, 5 Grudziadzka St., PL-87 100 Torun, Poland
}

\begin{abstract}
Optics as the enabling technology is applied in many applications of engineering, medicine, multimedia and conservation of cultural heritage. Most of these applications require close cooperation with the end user and often they enforce modification and enhancement of an optical tool. In the paper we show how optical metrology, specifically the application of digital image correlation method is implemented to two completely different tasks: performing preoperating tests of low cost building structures subjected to loading conditions which simulate the natural load e.g. introduced by the weight of snow and monitoring of canvas paintings for tracking humidity-induced deformations, which may appear in museum (or other location of a piece of art e.g. church).

The presented examples are the background for a general discussion on different measurement scenarios with application of DIC method, as well as the required enhancements and modifications which have been introduced.
\end{abstract}

Key words: hybrid experimental-numerical methodology, displacement/strain measurement, digital image correlation, civil engineering structure, canvas painting

\section{INTRODUCTION}

The mechanical quantities which carry most useful information about behavior and health of an arbitrary structure are displacements and strains [1]. Many methods provide this information point-wise only. This includes traditional strain gauges or fiber optics sensors. However full-field measurement methods providing displacement maps are more efficient and more compatible with data obtained from FEM or other numerical methods widely used in mechanical structures modeling. The most popular full-field methods are of optical methods. They are able to provide displacement data with different sensitivities, measurement ranges, spatial and temporal resolutions, complexity of measurement systems and capability to use them in laboratory or outdoor environments. The methods include coherent (holographic and speckle interferometry, grating interferometry) $[2,3]$ and noncoherent image-based methods (moiré fringe methods, structured light methods, digital image correlation) [4,5]. Sometimes, due to different global and local measurement requirements investigation of a structure is performed by a hierarchical system of full-field methods [6,7].

Analyzing a variety of demands coming from different end users it seems that in many cases 3D digital image correlation method is the very flexible and useful measurement tool $[5,8]$. It provides shape of a structure as well as in-plane and out-of-plane displacement fields $(\mathrm{u}, \mathrm{v}, \mathrm{w})$ which can be converted numerically into strains. 2D and 3D DIC provides a scalable field of view (FOV) and can deal with a wide range of the displacements. It achieves high accuracy of displacement measurement (up to 0,02 pixels) which, depending on the field of view and an array detector resolution, may refer even to submicrometer accuracy. Due to its flexibility and very simple measurement setup (two synchronized cameras and tripod or other geometry fixing element), 3D DIC is applied in many applications in the field of engineering, medicine, multimedia and conservation of cultural heritage. However most of these applications require close cooperation with the end user and often they enforce modification and enhancement of an optical tool. In order to reply to the end user needs DIC software should provide the capability to realize different measurement scenarios and provide the results in the form required by a customer. As the illustration to this statement we report on the applications of digital image correlation method to two completely different, interesting tasks. In the first scenario DIC is applied to

22nd Congress of the International Commission for Optics: Light for the Development of the World, edited by Ramón Rodríguez-Vera, Rufino Díaz-Uribe, Proc. of SPIE Vol. 8011, 80119R 2011 SPIE - CCC code: 0277-786X/11/\$18 - doi: 10.1117/12.915566 
performing pre-operating tests of low cost building structures subjected to loading conditions which simulate the natural load e.g. introduced by the weight of snow. In the second one DIC is used to monitor canvas paintings in order to track humidity-induced deformations, which may appear in museum or other location of a piece of art e.g. church.

\section{SCENARIO 1: 3D DIC FOR EXPERIMENTAL-NUMERICAL INVESTIGATION OF LOW COST BUILDING STRUCTURES}

\subsection{Background information}

The digital image correlation combined with FEM method can be a very powerful and efficient tool in civil engineering e.g. for investigation of building structures under different conditions. It is good alternative to expensive Structure Health Monitoring (SHM) systems as well as to conventional, strain gauges based measurements methods. The full-field information is especially advisable when the new, modern designs of low-cost building structures are investigated. The good example of such structures are graded metal plate structures (span up to $18 \mathrm{~m}$ ) which had been initially built temporarily for military applications [9] and were expected to be utilized in homogeneous climatic conditions over a period of a few months. However, a simple and fast technology of manufacturing and assembling called the attention of civilian investors, who adopted the technology to build bigger objects (span up to $30 \mathrm{~m}$ ) with the purpose of civil engineering applications and with a much longer utilization period (Fig.1a). The straightforward adaptation of the technology combined with parameters and purpose modification causes constructional issues particularly in regard to stability and load capacity. Moreover, the irregular metal plate surface is difficult to be numerically modeled and hence, the local loss of stability can occur in unexpected regions. Furthermore there is a lack of formal regulations (standards, instructions, guidelines), pertaining to methods of calculations of such structures. Simplified designs lead to crucial parameters being left out in the stability analysis which in consequence can lead to a catastrophic collapse.

The proposed investigation scenario for such structures is based on carrying out extensive laboratory tests on a 1:1 scale model in combination with FEM numerical analysis in order to provide a numerical model which is as close to the real structure, as possible This determines the hybrid experimental-numerical methodology [10,11], in which the laboratory measurements are performed to obtain a precise response to the simulated natural load. In the example below, the 1:1 scale model of the hall's arch was subjected to compression.

\subsection{Experiment and data analysis}

The specimen used in the experiment was a fragment of the metal-plate arch composed of four individual modules (Fig. 1b). Each module was made of a 1,25mm thick S355 steel cold-milled metal plate, which was composed of $3 \mathrm{~m}$ length segments. The segments were connected with M6 bolts. The cross-section of the metal-plate is presented in Figure 1c. The arch was fixed to the steel beam through a gusset, which was mounted with a dowel bar to the concrete foundation.

a)

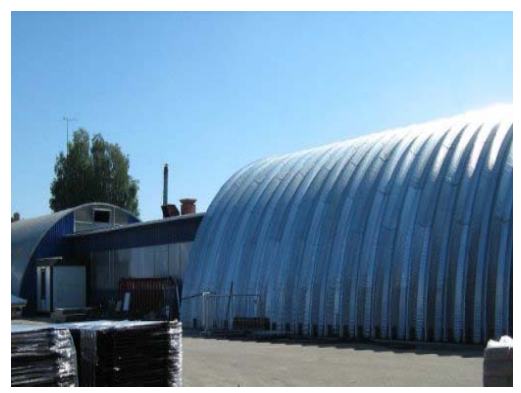

b)

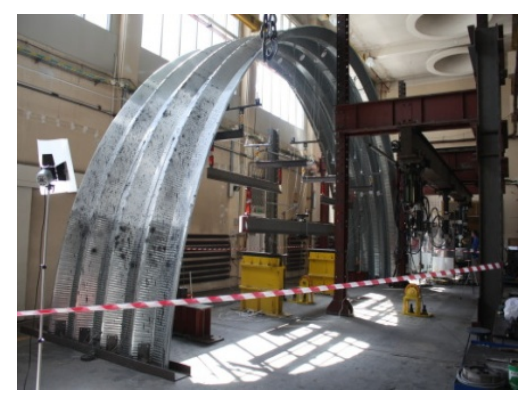

c)

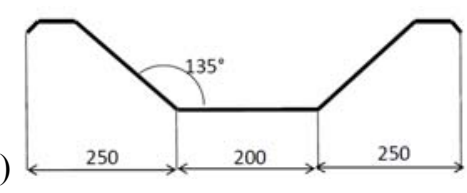

Figure 1. The real object and its physical model: a) a photo of a part of the warehouse complex, b) the experimentally investigated object and c) dimensions of the individual module. 
The loads were introduced in cycles. The aim of the test was to indicate critical load values and find bottlenecks of the FEM model. The test was carried out until the failure of the structure.

Altogether the 3D DIC sensor took more than 3000 measurements with a frequency of $1 \mathrm{~Hz}$. All control computers (force, 3D DIC, point-wise sensors) were time synchronized with each other. The synchronization of all systems facilitated data comparison procedures and ensured more reliable results.

a)

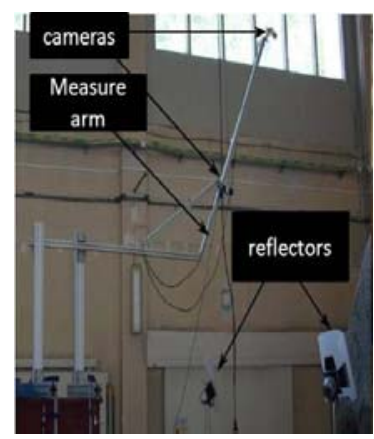

b)

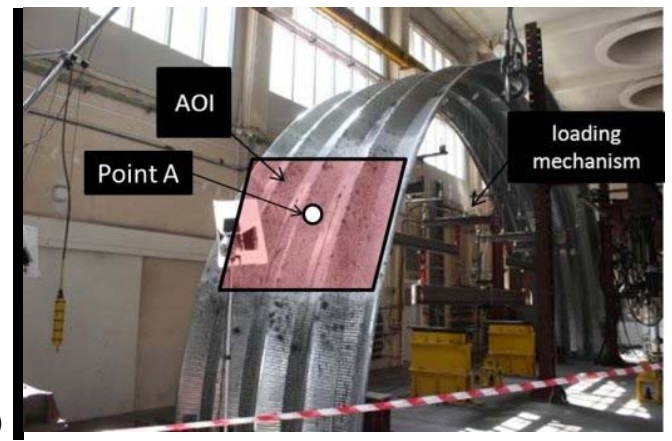

Figure 2. The experimental setup: a) view of the 3D DIC measuring arm, b) localization of the 3D DIC AOI and loading mechanism.

The 3D DIC setup and the object under examination are presented in Fig. 2. The $2 \mathrm{~m} \times 2 \mathrm{~m}$ field of view was located 2 meters above the floor (Fig. 2b), in the region, where the simplified FEM model analysis reveal the biggest displacements. The point A marked within the field of view was latter taken for comparison between DIC results and the measurement performed by a string sensor. The images were captured by two 2MPx AVT Stingray cameras equipped with Schneider Kreuznach $8 \mathrm{~mm}$ focal length lenses. The cameras were connected in a daisy chain mode to laptop via FireWire ExpressCard extension. In order to improve light conditions, two $650 \mathrm{~W}$ halogen reflectors were used. Because of the large FOV, the cameras were set apart $1,5 \mathrm{~m}$ from each other with an angle of $30^{\circ}$. The setup was installed on a scaffold made of aluminium constructional profiles (Fig. 2a). For the 3D DIC analysis, the VIC 3D software (by Correlated Solutions) has been used. The predicted accuracy for in-plane displacements is $50 \mu \mathrm{m}$ and for out-of-plane displacements is approximately $75 \mu \mathrm{m}$ (as scaled by the stereovision factor). Two exemplary sets of displacements maps calculated for two different loadings are shown in Fig. 3.

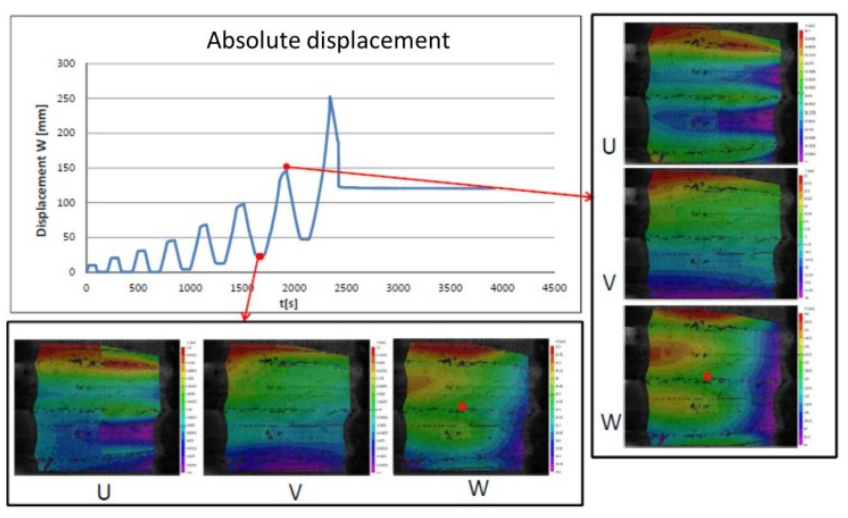

Figure 3. The exemplary results: displacement maps u, v, w and the plot of absolute displacement at point A.

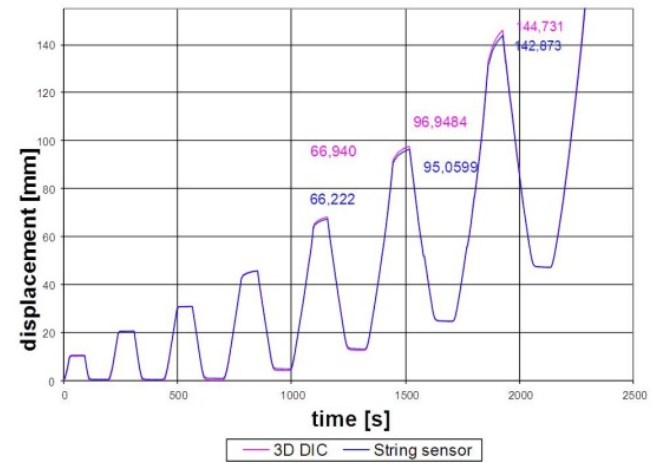

Figure 4. Comparison of the results obtained by different sensors: 3D DIC sensor and string sensor in point $\mathrm{A}$.

Displacements maps were monitored with a frequency of $1 \mathrm{~Hz}$ (using a commercial software package called Vic Snap). This feature is especially important when failure occurs simultaneously in a few regions, which are difficult to predict before the experiment.

In order to enable more reliable comparison of DIC measurement with the results obtained from the displacement string sensor, the absolute displacement value has been calculated. The string sensor was localized at the opposite side of the metal plate than the 3D DIC observation field. The localization of the sensor was therefore marked (to facilitate finding the right spot). Diagrams of the absolute displacements in point A obtained from both sensors are presented in Fig. 4. 
The analysis showed very good correlation between both sensors. The maximum discrepancy of measurements is less than $2 \%$. The small discrepancies could be caused by the fact that sensors were placed on the opposite sides of the metal plate.

The 3D DIC is a very attractive alternative to the standard point-wise measurement techniques. It is be much more efficient and effective to monitor big fields of view instead of installing sets of point-wise sensors.

The high correlation between the mentioned above measurement methods justified the utilization of results for the calibration of the FEM model. The obtained experimental data has been introduced into the simplified FEM model. Consequent detailed FEM model analysis aimed to determine the critical load with respect to the global buckling and the local loss of stability. The result from calculations is the critical load for the first (and the most probable) mode of buckling. The view of the FEM model subjected to critical load have been compared with the real image of the object under experimental load (Fig. 5).

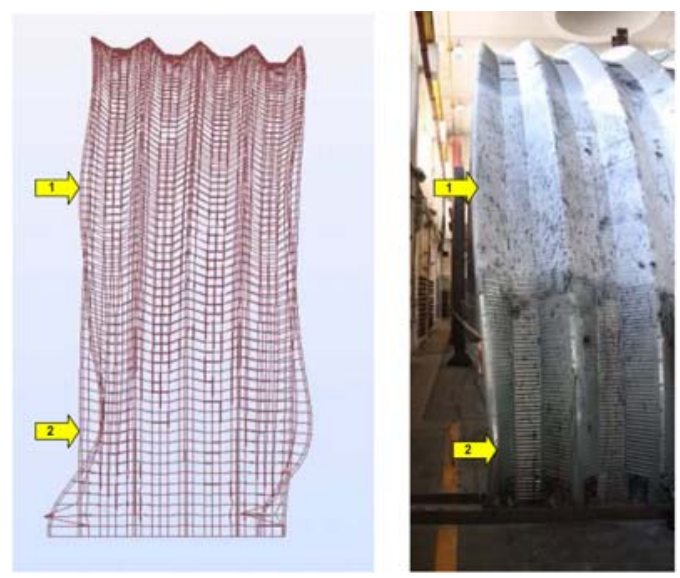

Figure 5. View on the model and view on the object during the experiment.

In the point 1 a buckling can be observed, while in point 2 the metal plate has been broken as a result of the loss of stability. During the experiment, the loss of stability occurred for the $37.4 \mathrm{kN}$ summarized load, which matches the numerically calculated value very well $(36.6 \mathrm{kN})$. This very good corresp.ondence between the FEM model and the real test object allows to conclude that the model calibration was correct.

\section{SCENARIO 2: 3D DIC FOR CANVAS PAINTINGS INVESTIGATION}

\subsection{Background information}

Canvas paintings are complex, multilayer structures composed of hygroscopic materials with different properties: canvas support, glue, ground, oil paint and varnish [12]. In many cases historical canvases are repaired and patched with materials which have different, from the original, physical properties. Therefore, when environmental conditions are changing, inner stresses appear within the composite material [13]. The stresses can cause cracking and delamitation of a canvas painting. To monitor and eventually minimize these effects in environments with rapidly changing conditions, (like exhibitions which are visited by many people), it is recommended to apply a full field displacement/strains measurement system. However it is also necessary to interpret properly the results of the measurements and use them as the feed back signal for control of a local environment.

\subsection{Experiments and data analysis of model canvas paintings}

To assess the applicability of 3D DIC in monitoring of canvas paintings, the tests at model canvas were performed [14]. In the 3D DIC setup (Fig. 6) two CANON EOS 5D Mark II (5616 x 3774 pixels) cameras equipped with 28mm CANON lenses had been used. The setup and the model canvas were mounted at an optical table to provide mechanical stability. The field of view (FOV) was $0.6 \mathrm{~m} \times 0.4 \mathrm{~m}$ and the expected accuracy of displacement measurement was $20 \mu \mathrm{m}$. In order to ensure sufficient light condition, a $650 \mathrm{~W}$ halogen lamp was used. 


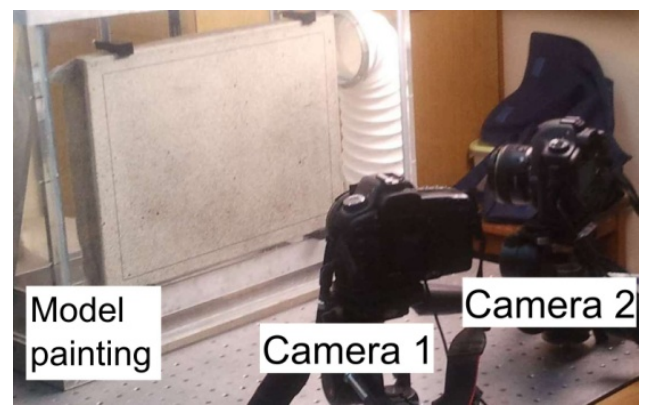

Figure 6. 3D DIC setup with model painting.

The model canvas with dimensions: $40 \mathrm{~cm} \times 30 \mathrm{~cm}$ and with different types of patches distributed within FOV were used as the object of examination were the object. The canvas were painted with a stochastic pattern appropriate for the DIC method. During the test, the relative humidity level was changed in the range from $30 \%$ to $70 \%$ RH in a custom designed airtight climate chamber. DIC data (a pair of images) together with the relative humidity and temperature values were captured every 20 seconds.

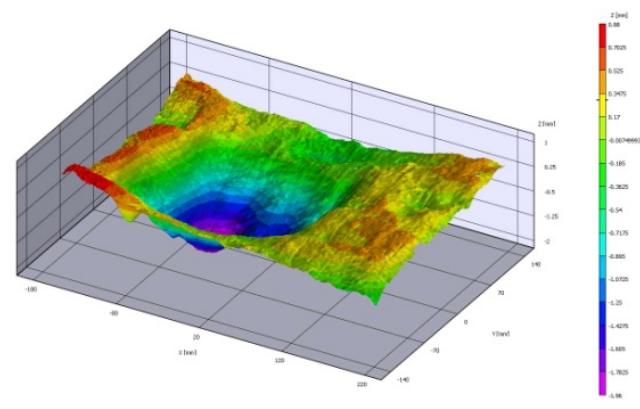

Figure 7. The map of global shape of the painting before test P-V: $2,84 \mathrm{~mm}$.

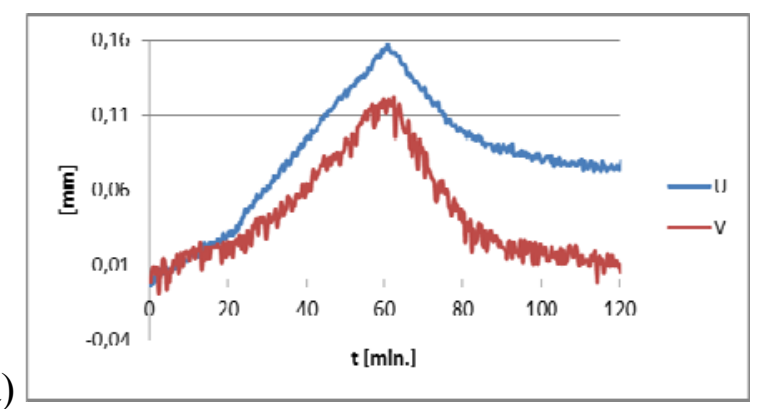

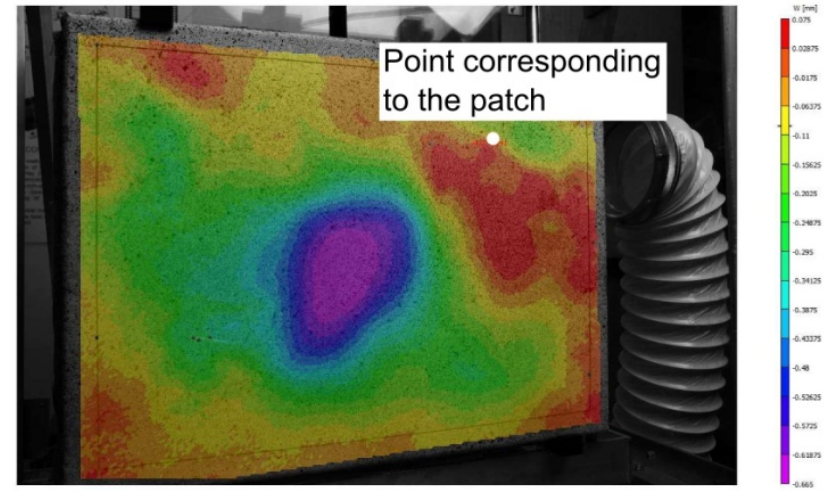

Figure 8. An exemplary map of out-of-plane displacement w(x,y) with the marked point corresponding to one of the patches.

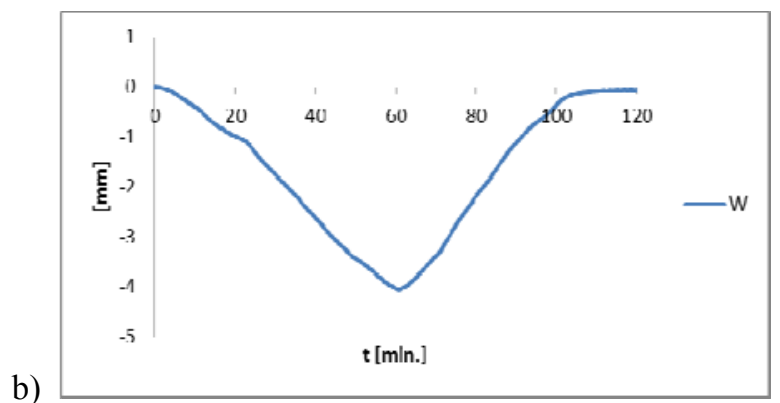

Figure 9. Monitoring of displacements in the point corresponding to one of the patches (Fig.8): a) u,v and b) w.

After the test, the initial shape of the model (Fig. 7) and the u,v,w (Fig. 8) displacement maps were calculated. For the exemplary points corresponding to the patches the plots of all displacements in the function of time have been analysed. The exemplary plots for in-plane displacements $u, v$ and out-of-plane displacement w are shown in Fig. 9a and 9b. It is clearly seen that the values of out-of-plane displacements are one order of magnitude bigger than the in-plane displacements. However the signal-to-noise ratio achieved even for the small values of displacements is sufficient to monitor the influence of changing environmental conditions. 


\subsection{Experiments and data analysis of painting with real texture}

As mentioned above the 3D DIC method requires stochastic pattern which can be difficult to provide in real art objects. However we can expect that a variety of paintings has its own texture which can be treated as a naturally stochastic pattern (Fig. 10). Of course the level of the measurement confidence in a given pixel will depend on the presence of the local intensity variations. In the areas with constant intensity the correlation between two images cannot be determined and the regions are masked out (Fig. 11). Also the local level of confidence interval (for each match) [15] can be used for determination of local uncertainty of the measurement. The tests of the canvas painting with natural texture had been performed in the same setup as with the model object, however the displacement maps were introduced mechanically.

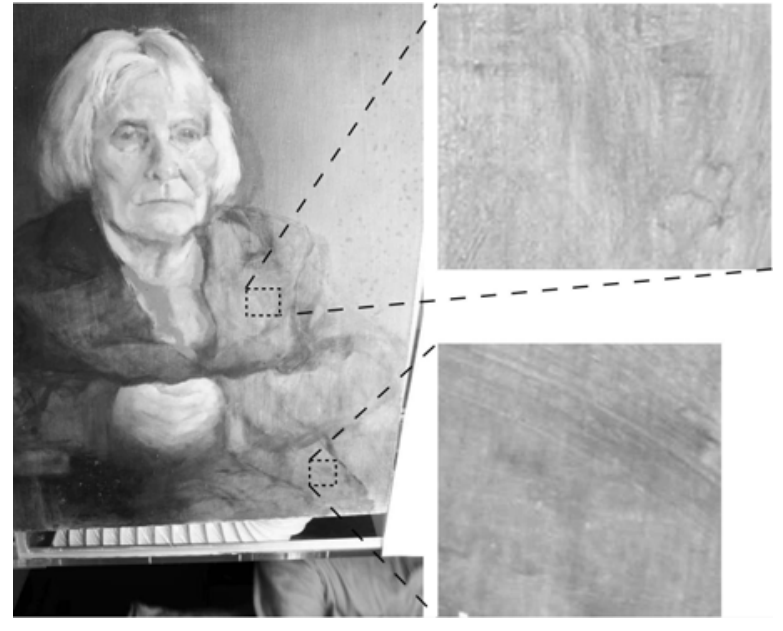

Fig. 10 The investigated object with indication of the local texture.

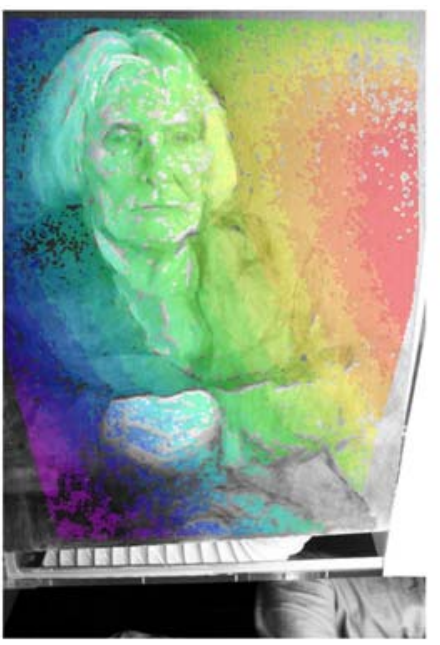

a)

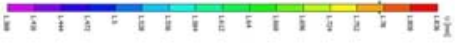

Figure 12. The maps of displacements

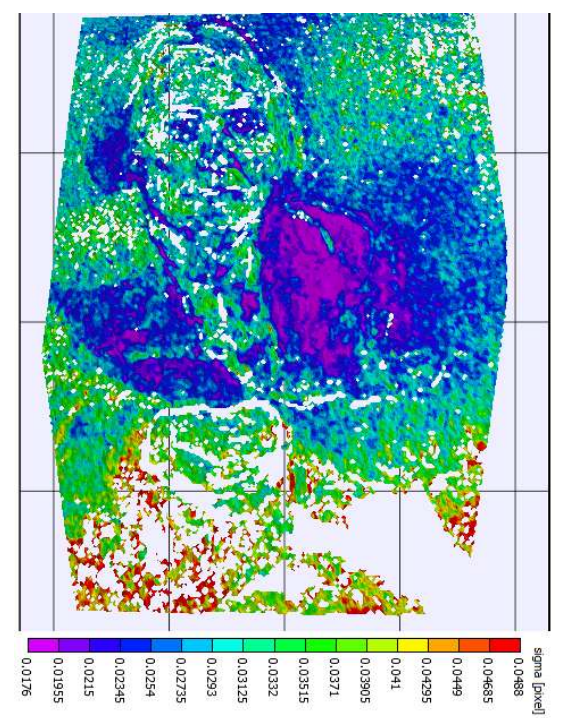

Fig. 11. The map of the confidence interval for the match at each point, in pixels.
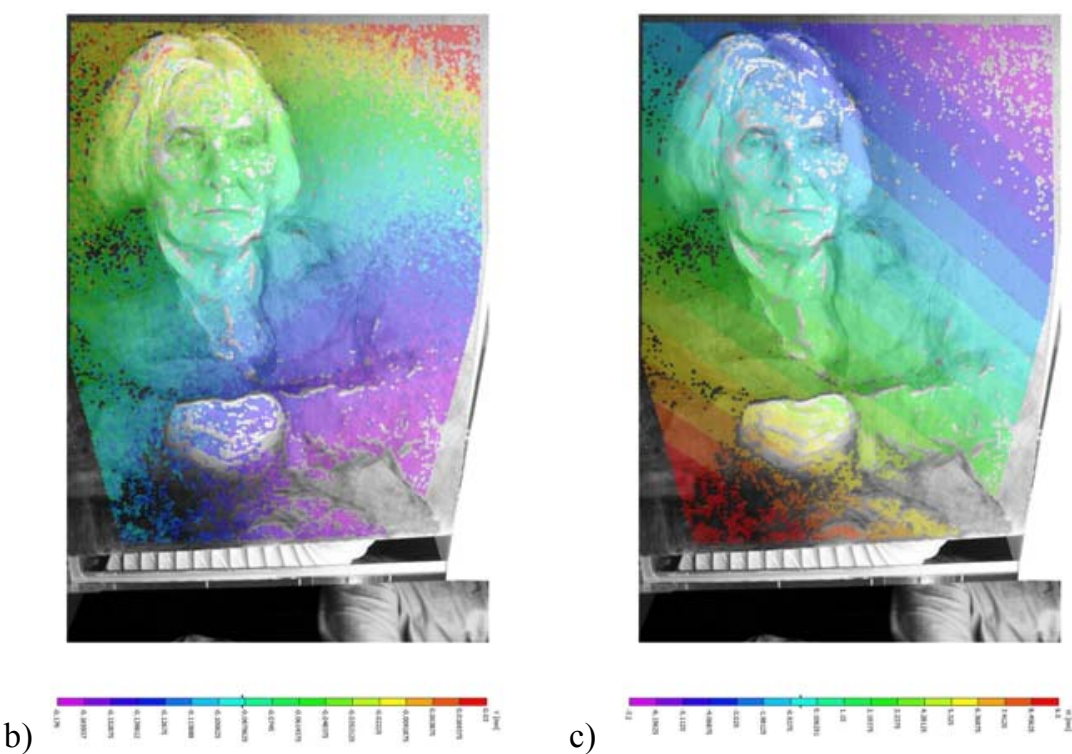

c)

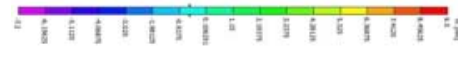


The displacement maps are well determined in the areas where the measurement confidence level is above the set threshold. This proves initially the capability of 3D DIC method to be applied directly for real canvas paintings investigation. Of course at first the texture of paintings have to be tested for the level of measurement confidence, therefore the software should allow for quick test of this quantity, setting the chosen confidence threshold and eventually showing the area where high uncertainty of the measurement is expected.

\section{CONCLUSIONS}

Based on two very different measurement scenarios the great flexibility and wide applicability of 3D DIC method and instrumentation is shown. Although all applications use the same output quantities i.e. shape and displacements they are addressed to different end users and need different supporting tools, user interfaces and visualization.

The most often applications of DIC are experimental mechanics and material engineering, therefore its extension to 1:1 scale model examination in combination with FEM numerical analysis (as shown in Scenario1) is straightforward. However as it is not present in the standardized tests of mechanical structures and therefore it has to be supported by the measurements performed by the officially recognized tools as strain gauges or string sensors. This lack of procedures which are fully recognizing DIC method as the accepted measurement tool is the biggest obstacle in its wide implementation in the areas where safety and health of engineering structures are considered.

The second Scenario is connected with safety and quality of conservation of artworks. It has been shown that 3D DIC is well suited for canvas paintings measurements, however in order to address the real artworks their natural texture should be used. It is possible in many cases but the measurement uncertainty may be lower when compared with the measurements performed at stochastic artificial texture. Therefore it is necessary to monitor the local confidence of a measurement and modify the testing methodology accordingly. In future it is possible to use three color channels for separate measurements and compose displacement maps based on the results obtained for a given pixel in the highest confidence channel.

In different scenarios and applications it is necessary to combine the displacement and shape data with other experimental data (e.g. temperature maps) or/and numerical results in order to create hybrid tool providing extensive knowledge about an investigated object.

\section{ACKNOWLEDGEMENTS}

The financial support from the project "Health Monitoring and Lifetime Assessment of Structures"-MONITPOIG.0101.02-00-013/08-00 from the EU Structural Funds in Poland and from the statutory work of Warsaw University of Technology are gratefully acknowledged.

\section{REFERENCES}

[1] A.S. Kobayashi, "Handbook on experimental mechanics", SEM, 1993

[2] T. Kreis, "Holographic interferometry: principles and methods", Akademie Verlag, Berlin, 1996

[3] R.S. Sirohi (ed), "Speckle metrology”, Marcel Dekker, New York, 1993

[4] K. Patorski, "Handbook of the Moire Fringe technique", Elsevier, Amsterdam,1993

[5] M. Sutton, J-J Orteu, H. Schreier, "Image correlation for shape, motion and deformation measurements", Springer, 2009

[6] M Kujawinska et al. "Remote online monitoring and measuring system for civil engineering structures", Proc. SPIE, vol. 7389, 738904-1-10, 2009.

[7] M. Kujawińska, R. Sitnik, G. Dymny, M. Malesa, K. Malowany, D. Szczepanek, „Hierarchical, multitasks optical system for health monitoring of civil engineering structures", Proc. SPIE, vol. 7387:738721, 2010

[8] B. Pan "Recent Progress in Digital Image Correlation" , Experimental Mechanics, online First, DOI:10.1007/s 11340 -010-9418-3, 2010

[9] www.tgbuildings.com (in Polish) 
[10] K-H. Laermann, "Hybrid techniques in experimental mechanics", chapter in "Optical methods in experimental solid mechanics", Springer, WienNewYork, 2000.

[11] M. Malesa, M. Kujawińska, D. Szczepanek, A. Swiercz, P. Kolakowski, „Monitoring of Civil Engineering Structures Using DIC Technique”, Book of Abstracts ICEM14, pp 31014, 2010

[12] M. F. Mecklenburg, "The structure of Canvas Supported Paintings", Preprints of the Int. Conference on Painting Conservation - Canvases: Behaviour, detoration and Treatment. 119-155, 2005

[13] D. Erhardt, C.S. Tumosa, M.F. Mecklenburg, "Applying science to the question of museum climate”, Museum microclimates: contribution to the Copenhagen conference, 11-18, 2007

[14] M. Malesa et al., "Application of Digital Image Correlation (DIC) for tracking deformations of paintings on canvas”, Proc. SPIE, vol. 8084, 8084L-1, 2011

[15] http://www.correlatedsolutions.com 